\title{
Fusarium Head Blight Severity and Deoxynivalenol Concentration in Wheat in Response to Gibberella zeae Inoculum Concentration
}

\author{
J. M. Stein, L. E. Osborne, K. D. Bondalapati, K. D. Glover, and C. A. Nelson
}

Plant Science Department, South Dakota State University, Brookings SD 57007.

Accepted for publication 26 February 2009.

\begin{abstract}
Stein, J. M., Osborne, L. E., Bondalapati, K. D., Glover, K. D., and Nelson, C. A. 2009. Fusarium head blight severity and deoxynivalenol concentration in wheat in response to Gibberella zeae inoculum concentration. Phytopathology 99:759-764.

The relationship between inoculum dose and resulting disease levels and deoxynivalenol (DON) accumulation in the Fusarium head blight (FHB) of wheat pathosystem was examined under controlled conditions. Greenhouse-grown spring wheat plants were inoculated at flowering with suspensions that varied in Gibberella zeae macroconidia concentration. The spikes were bagged for $72 \mathrm{~h}$ to promote infection and plants were then kept under ambient greenhouse conditions and disease allowed to develop. Spikes were rated at 15 days after inoculation for disease inci-

mathematical relationship between inoculum dose and the (i) disease metrics or (ii) DON concentration. Both disease incidence and severity were found to increase sharply in relation to inoculum concentration until an asymptote was reached. In both instances, a negative exponential function was found to best explain this relationship. By contrast, DON concentration in both grain-only and whole-spike tissues increased with additional inoculum. These relationships were best explained with linear functions for both sample types, although DON accumulation increased at a greater rate in whole-spike tissue. The functions were evaluated further using data collected from unrelated field studies and, although not particularly consistent, provided reasonably accurate predictions in growing seasons when the environment was only moderately favorable for FHB
\end{abstract} dence and severity, removed from the greenhouse, and dried. DON concentration was determined in grain-only and whole-spike samples for each inoculation treatment. Regression analysis was used to evaluate the
Additional keywords: mycotoxin.
Fusarium head blight (FHB), caused primarily by Gibberella zeae (Schwein.) Petch (anamorph: Fusarium graminearum Schwabe), is a major fungal disease of wheat and barley $(9,13-$ $15,20,31)$. G. zeae is homothallic and readily produces both ascospores and macroconidia in nature. This fungus overwinters within plant residues (e.g., wheat, maize, and others) as both mycelia and perithecial initials which mature to produce ascospores when environmental conditions are favorable $(5,8,23)$. Macroconidia, hereafter referred to as 'conidia', are also readily produced from mycelia present on the surface of these residues. Ascospores are the only propagules that are actively discharged; however, both types can become airborne in large numbers $(6,20,25,26)$.

Economic losses associated with FHB occur because of the direct blighting of floral tissues (reduction in grain number), from the disruption of grain fill (shriveled kernels leading to lower test weight), and, most importantly, through the contamination of grain with mycotoxins, primarily deoxynivalenol (DON). Losses due to FHB can be substantial and have severely impacted producer regions such as the Northern Great Plains of the United States. The most common strategies utilized to reduce losses from FHB are the planting of less-susceptible cultivars, application of fungicide at flowering, and crop rotation or residue management to limit the level of in-field inoculum.

The relationship between $G$. zeae inoculum dose and resulting disease in wheat has been examined under controlled conditions for conidia alone (1) and conidia and ascospores (29). In both studies, the disease metric (i.e., incidence or severity) measured increased sharply in proportion to spore concentration until a

Corresponding author: J. M. Stein; E-mail address: jeff.stein@ sdstate.edu

doi:10.1094/PHYTO-99-6-0759

(c) 2009 The American Phytopathological Society maximum was reached where additional inoculum did not substantially increase the level of disease. In the latter study, both propagules were also found to be comparable in their ability to infect wheat and initiate FHB. Mathematical functions describing these relationships were not generated in either study and the relationship between inoculum dose and DON concentration in the grain has yet to be thoroughly examined $(16,30)$. By having a better understanding of the role inoculum concentration plays in disease development and DON accumulation, the effectiveness of risk advisory systems could potentially be increased, resulting in a lowered impact of this disease. That stated, the primary objective of this study was to mathematically characterize the relationships between $G$. zeae inoculum concentration on wheat spikes and the resulting (i) disease development (FHB incidence and severity) and (ii) DON concentration in grain-only and wholespike samples. A secondary objective was to test the effectiveness of these models to predict disease and grain DON concentration in field trials based solely on spike-borne inoculum loads.

\section{MATERIALS AND METHODS}

Plant inoculations. Greenhouse experiments were conducted in 2006 and 2007 to determine the effects of varying G. zeae inoculum dose on FHB disease and DON concentration in spring wheat. Seed of the FHB-susceptible hard red spring wheat line SD3854 were planted at 10 seeds/pot in 2.9-liter plastic pots using a commercial potting mix (Premier ProMix BX; Premier Horticulture Ltd., Quaker, PA). Natural sunlight was supplemented with 400-W high-pressure sodium lamps to achieve a 16-h day and 8-h night cycle. Temperatures were kept between $24^{\circ} \mathrm{C}$ (night) and $29^{\circ} \mathrm{C}$ (day). Plants were fertilized every 3 to 4 weeks with $20 \mathrm{~g}$ of controlled-release fertilizer (Multicote 4; Haifa Chemicals Ltd, Altamonte Springs, FL). Plants in individual pots were considered 
to be experimental units, unless otherwise noted, and the experiment was repeated three times (runs).

Inoculum concentration treatments included suspensions of 0 , $100,500,1,000,5,000,10,000,50,000$, and 100,000 conidia/ml in sterile, deionized water $\left(\mathrm{sdH}_{2} \mathrm{O}\right)$ with $0.125 \%$ ( $\left.\mathrm{vol} / \mathrm{vol}\right)$ polysorbate 80 (Tween 80 ). Conidial suspensions of ' $\mathrm{Fg} 4$ ', a highly aggressive and toxigenic isolate of $G$. zeae, were generated by washing 14-day-old mung bean agar cultures with $2 \mathrm{ml}$ of $\mathrm{sdH}_{2} \mathrm{O}$ Tween. Conidia were then dislodged using a sterilized glass rod. The suspension volume was increased to $\approx 200 \mathrm{ml}$ and filtered through six layers of sterile cheesecloth to remove larger sections of mycelium and other debris. Conidial concentration was enumerated microscopically and adjusted to $1.0 \times 10^{5}$ conidia $/ \mathrm{ml}$ using additional $\mathrm{sdH}_{2} \mathrm{O}$-Tween. Serial dilutions were then prepared as necessary. The final concentration of each was verified microscopically and was also plated on acidified potato dextrose agar media to verify inoculum viability. In all cases, $>85 \%$ of the conidia germinated.

When a majority of the main-tiller spikes in each pot (6+) were between Feekes 10.52 and 10.53 (10), they were inoculated by spraying the appropriate conidial suspension using a trigger-pump misting sprayer until spikes were saturated. The spikes were allowed to air dry for $15 \mathrm{~min}$, then covered with plastic, zippertype bags (76 by $127 \mathrm{~mm}$ ) to promote high-humidity conditions and incubated for $72 \mathrm{~h}$ on the greenhouse bench. After the incubation period, the bags were removed and the pots retained on the bench. At 15 days post inoculation, the number of diseased and total spikelets was enumerated for each treated spike in a pot. Disease incidence (number of diseased spikes/total) and average severity (number of diseased spikelets/total) were calculated for each pot.

Mycotoxin analysis. Following disease ratings, spikes from each pot were removed from plants, put into brown paper sacks, and placed in an oven at $30^{\circ} \mathrm{C}$ for $72 \mathrm{~h}$ to dry. Individual spikes from each plant were hand threshed, and both grain and chaff were separately retained. The grain samples included all identifiable kernels, including those shriveled due to direct colonization by or indirect disruption from infection by G. zeae. The chaff samples included the rachis, rachilla, glumes, palea, lemma, and awns. Portions of the peduncle were discarded and weights were recorded for both fractions from each pot.

Based on component weights of tissues from each pot, samples were prepared for grinding and mycotoxin analysis. Two types of tissue samples were ultimately analyzed: grain-only (GO) and whole-spike (WS) samples. The latter consisted of the recombined grain and chaff portions from an individual pot. Plants from the higher-spore-concentration treatments occasionally produced less grain than the 5-g mass required for toxin analysis. In such cases, samples were pooled from two or more replicate pots for toxin analysis. The disease metrics for such samples were recalculated using the combined data set from the combined pots. There was a minimum of three replicate samples per concentration for each GO and WS sample type. Tissues were ground using a laboratory sample mill (UDY Cyclone; UDY Corporation, Ft. Collins, CO) to pass a $0.5-\mathrm{mm}$ screen. Samples were analyzed for DON using gas chromatography-mass spectrophotometry (GC-MS) with a lower detection limit of $0.5 \mu \mathrm{g} / \mathrm{kg}$, at the North Dakota Veterinary Diagnostic Laboratory (Fargo, ND). Samples that had less than the detectable limit of mycotoxin were assigned a value of $0.25 \mu \mathrm{g} / \mathrm{kg}$ (one-half the detection limit), as is typically done with such data.

Data analysis and model development. It was determined experimentally during the first run that spikes received, on average, $0.25 \mathrm{ml}( \pm 0.06 \mathrm{ml})$ of liquid when inoculated, or $\approx 25 \%$ of the conidia in $1.0 \mathrm{ml}$ of the suspension. Therefore, the values used for spore concentration in the analyses were adjusted to represent the estimated number of conidia being applied to a spike based on this proportion (e.g., 25,000 conidia/spike for the
100,000 conidia/ml treatment). This estimated number of conidia applied to a spike is hereafter referred to simply as the 'inoculum concentration'.

Linear and nonlinear regressions were used to examine relationships between inoculum concentration and the resulting disease metrics or DON accumulation. These analyses were conducted with the statistical package R (v. 2.7.2) (24) using the nonlinear mixed effects models (NLME) package (11). Negative exponential and linear models were fit for the disease metrics and mycotoxin concentrations, respectively. In all cases, parameters for each function (e.g., rate of increase) were treated as random effects with the data being partitioned by run over which the random effects varied.

Once the range of $G$. zeae propagules present on wheat spikes under field conditions was determined (see below), the parameter estimates were recalculated using this range and the results were compared. Specifically, because the maximum number of G. zeae propagules enumerated in the field studies was $\approx 2,500$ propagules/spike, new parameter estimates were calculated based on results from the greenhouse study data but using the range of propagules identified in the field studies ( 0 to 2,500 instead of 0 to 25,000). These estimates are hereafter referred to as being from the field and greenhouse inoculum ranges, respectively.

Evaluation of models under field conditions. Data collected from field studies were used to test the ability of these models to predict disease and DON based solely on inoculum concentration. These studies were conducted on the South Dakota State University Agronomy Farm in Brookings during the 2003-05 and 2007 growing seasons as part of two multistate collaborative efforts and are hereafter referred to as 'EDM0x' and 'Residue0x' for FHB epidemiology and residue management studies, respectively. In both cases, ' $\mathrm{x}$ ' is the year the study was conducted (e.g., EDM05 in 2005). In all studies, a FHB-susceptible cultivar of hard, red spring wheat (either 'Norm' or 'Russ') was grown under standard cultural practices for the region, and the level of inoculum in individual plots was manipulated using varying levels of naturally colonized maize residue $(16,19)$. No additional inoculum was applied, nor was misting employed to increase the probability of infection. Disease metrics and DON concentration in the grain were determined in a manner similar to that used in the aforementioned greenhouse studies, except on a field plot scale as detailed elsewhere $(17,18)$.

To determine the inoculum intensity for a plot, wheat spikes were collected daily during the peak period of flowering $(\approx 7$ days) and washed to dislodge spores. This suspension was then concentrated and plated onto semiselective media. The number of CFU that were of the G. zeae type was enumerated after 7 days. The proportion of ascospores versus conidia was not determined. A subset of the putative isolates was grown out further to determine the species. In all cases, $>90 \%$ of the CFU counted were confirmed to be $G$. zeae. Additional details of this procedure can be found elsewhere $(7,17,18)$.

The expected disease incidence, severity, and concentration of DON in the grain were then calculated for all replicate plots in each field study using the functions described above with both the field and greenhouse inoculum ranges. Because plants in the field probably received inoculum over multiple days instead of a single dose as in the greenhouse study, the mean number of CFU/spike over the period of susceptibility was used in the calculations.

\section{RESULTS}

Relationship between inoculum and disease. Under controlled conditions, both the disease incidence and severity increased with the number of G. zeae conidia applied to susceptible wheat spikes. In both cases, a negative exponential function explained this relationship, although the rate of increase and asymptote (average maximum) differed between metrics (Fig. 1; 
Table 1). Disease incidence approached $100 \%$ of the spikes in some replicate pots with as few as 25 conidia/spike (i.e., the 100 -conidia/ml inoculum concentration), although it was determined that $\approx 600$ conidia/spike was required to reach $99.9 \%$ of the average asymptote (functional maximum). In comparison, $\approx 12,300$ conidia/spike were required to reach this same level for the disease severity. In other terms, 20.5 times the number of conidia per spike were required to approach the maximum for disease severity compared with incidence when susceptible wheat plants were kept under favorable conditions for infection and symptom development. The results varied within and between experimental repeats for both metrics, especially at the lower concentrations (e.g., $<5,000$ conidia/spike). A low level of disease developed in some of the control spikes, indicating a background level of inoculum in the greenhouse.

Relationship between inoculum and DON. As with the disease metrics, the DON present in both the GO and WS samples increased with respect to inoculum concentration (Fig. 2). However, the function for this relationship was linear and not exponential, indicating a different response to inoculum concentration. The rates of increase (slopes) for the two sample types using the full inoculum range were 0.002 and $0.004 \mu \mathrm{g} / \mathrm{kg}$ DON per conidium per spike for the GO and WS, respectively (Table 1). DON concentration increased in the WS samples (with respect to inoculum concentration) at $\approx 2.5$ times the rate of the GO samples. The results for DON concentration also varied within and between runs for both sample types; however, this variability was greatest at the higher spore concentrations and not the lower ones as with the disease metrics. There were no instances where DON was detected in the absence of disease symptoms.

Field evaluation of models. As with the greenhouse studies, the disease metrics and grain DON concentration in the field studies all tended to increase in relation to the quantity of G. zeae inoculum present on the spikes during the period of peak flowering (Figs. 3 and 4, respectively). The minimum and maximum mean number of $G$. zeae propagules present on wheat spikes was 40 and 2,162 spikes/day, and the mean number of propagules per spike per day for all plots differed substantially between seasons (e.g., 71 for EDM03 versus 1,115 for EDM04).

When the expected values for incidence, severity, and DON were calculated and compared with the observed values, none of the models described was consistently able to accurately predict the resulting level (Figs. 3 and 4; Table 2). The functions for disease incidence tended to overestimate the predicted level at

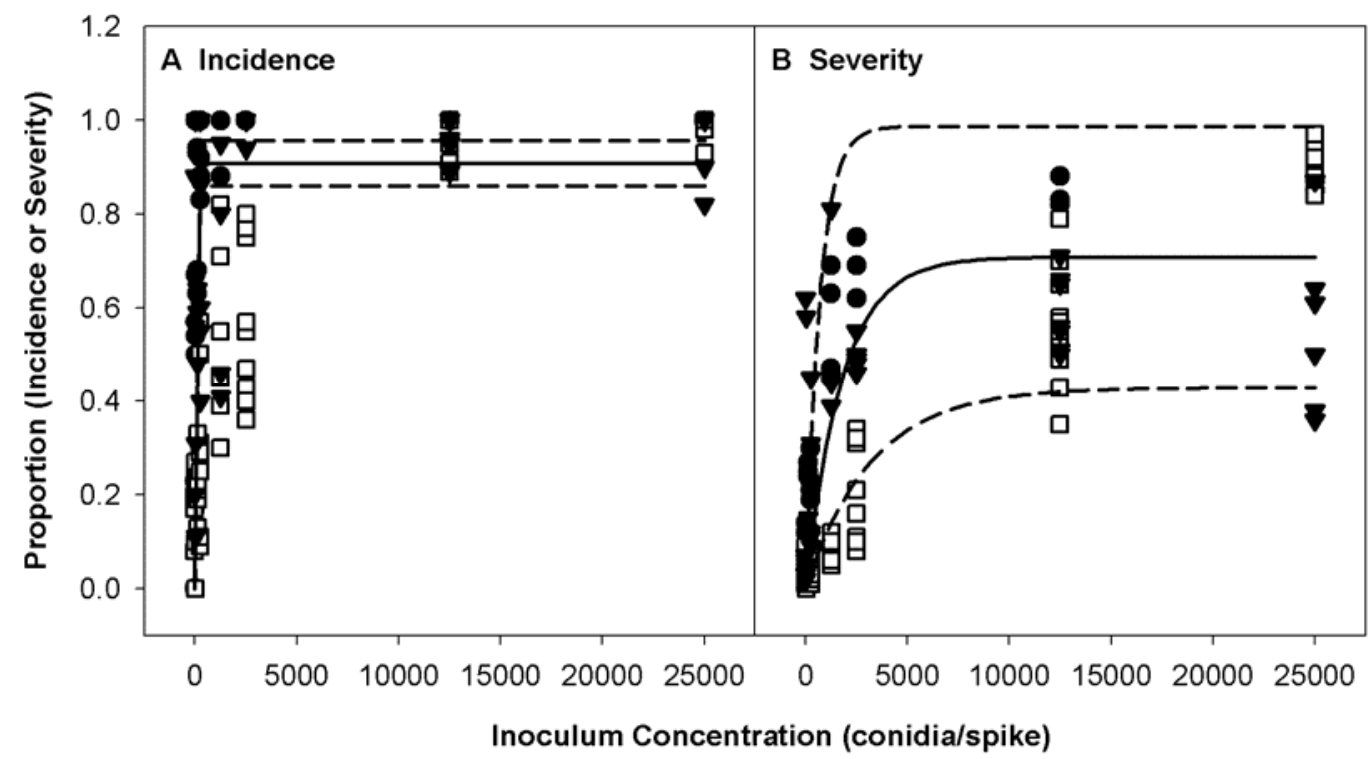

Fig. 1. Concentration of Gibberella zeae inoculum applied to a susceptible wheat line and the resulting disease A, incidence or B, severity for experimental runs one ( $\square$ ), two $(\bullet)$, and three $(\boldsymbol{\nabla})$ of the greenhouse study. Nonlinear regression lines represent the predicted mean value (solid) and $95 \%$ confidence intervals (dashed).

TABLE 1. Model functions and parameter estimates predictive of Fusarium head blight incidence and severity, and deoxynivalenol (DON) accumulation in the grain-only and whole-spike samples based on Gibberella zeae inoculum dosage (conidia/spike) applied to a susceptible wheat line

\begin{tabular}{|c|c|c|c|c|c|c|}
\hline \multirow[b]{2}{*}{ Response variable } & \multirow[b]{2}{*}{ Model $^{\mathrm{x}}$} & \multirow[b]{2}{*}{ Inoculum $^{\mathrm{y}}$} & \multicolumn{4}{|c|}{ Parameter estimates (standard deviation) } \\
\hline & & & $L$ (asymptote) & $k$ (rate) & $a{\text { (intercept })^{\mathrm{z}}}$ & $b$ (slope) \\
\hline \multicolumn{7}{|l|}{ Disease metrics } \\
\hline Incidence & Neg. Exp. & GH & $0.908(0.025)$ & $0.012(0.027)$ & $\ldots$ & $\ldots$ \\
\hline \multirow[t]{2}{*}{ Severity } & Neg. Exp. & GH & $0.708(0.142)$ & $0.001(0.001)$ & $\ldots$ & $\ldots$ \\
\hline & & Field & $0.555(0.051)$ & $0.002(0.001)$ & $\ldots$ & $\ldots$ \\
\hline \multicolumn{7}{|l|}{ DON concentration } \\
\hline Whole-spike & & Field & $\ldots$ & $\ldots$ & $7.735(3.656)$ & $0.016(0.006)$ \\
\hline
\end{tabular}


low-inoculum concentrations and the asymptote was best predicted when fit to the function consisting of the parameter estimates generated from the full greenhouse inoculum range. By contrast, the function for disease severity performed much better overall, with the greenhouse- and field-generated parameter estimates being most accurate at the low and high inoculum ranges, respectively. The functions developed to predict DON levels were accurate at the low-inoculum concentrations and in situations where the final DON concentration was $<5 \mu \mathrm{g} / \mathrm{kg}$ (Fig. 4). The DON level was greatly underestimated by these functions in the EDM04 study (Fig. 4; Table 2).

\section{DISCUSSION}

The primary objective of this study was to mathematically characterize the relationships between spike-borne G. zeae inoculum and the resulting development of disease and DON accumulation in a susceptible wheat line. It was determined experimentally that disease incidence and severity were best explained with a negative exponential function under both greenhouse and field conditions. The level of disease rapidly reached an asymptote for both metrics, with addition of inoculum having no further effects. In contrast, DON accumulation in the ranges of inoculum tested was best explained with a linear function where the concentration continued to increase with additional inoculum. However, it should be noted that this response would probably also follow a negative exponential or similar function in relation to inoculum dose because, at some point, the curve would be saturated (e.g., DON at 1,000,000 $\mu \mathrm{g} / \mathrm{kg}$ ).

Functions developed from the greenhouse studies for disease and DON were not particularly effective at predicting events in the field. This was true even when the parameter estimates were recalculated based on the range of inoculum normally present under field conditions (i.e., 0 to 2,500 versus 0 to 25,000 conidia/spike). Specifically, disease incidence was overestimated at the lower inoculum concentrations while disease severity and DON were underestimated. Several factors may explain why these models performed marginally under field conditions. First,

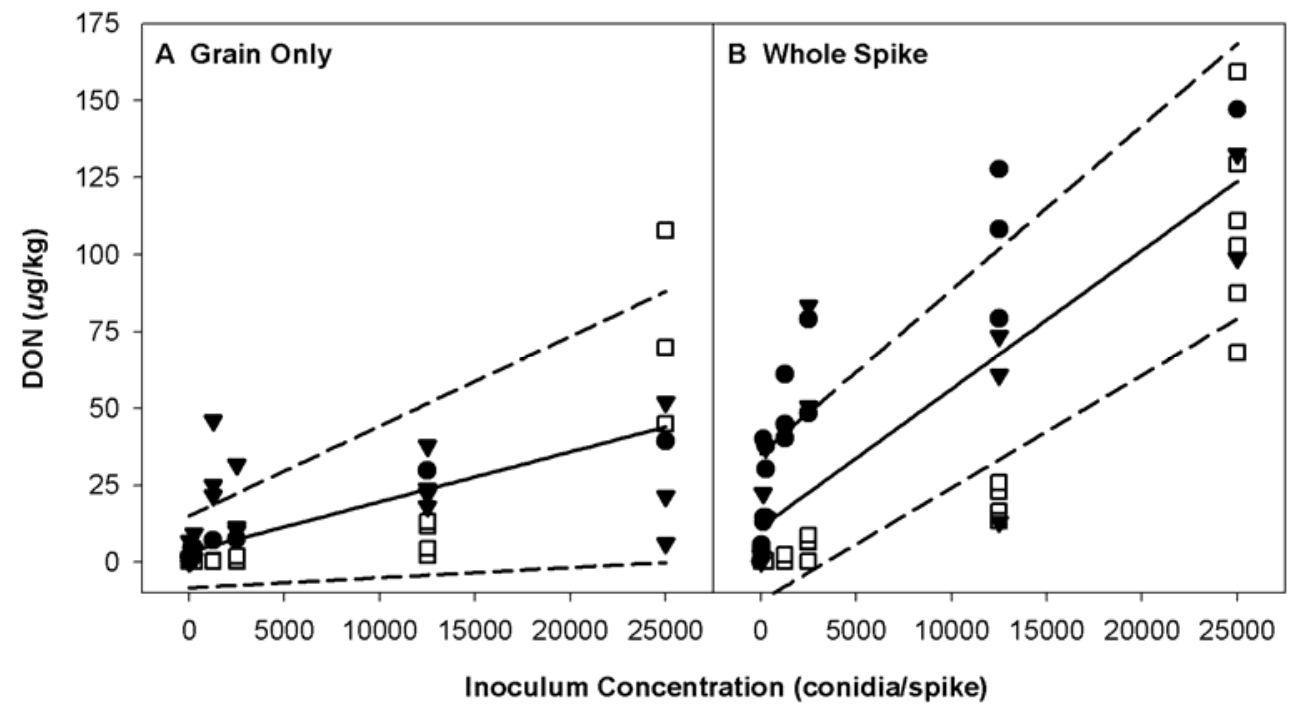

Fig. 2. Concentration of Gibberella zeae inoculum applied to a susceptible wheat line and the resulting deoxynivalenol (DON) concentration in the A, grain-only or B, whole-spike samples for experimental runs one $(\square)$, two $(\bullet)$, and three $(\boldsymbol{\nabla})$ of the greenhouse study. Linear regression lines represent the predicted mean value (solid) and $95 \%$ confidence intervals (dashed).

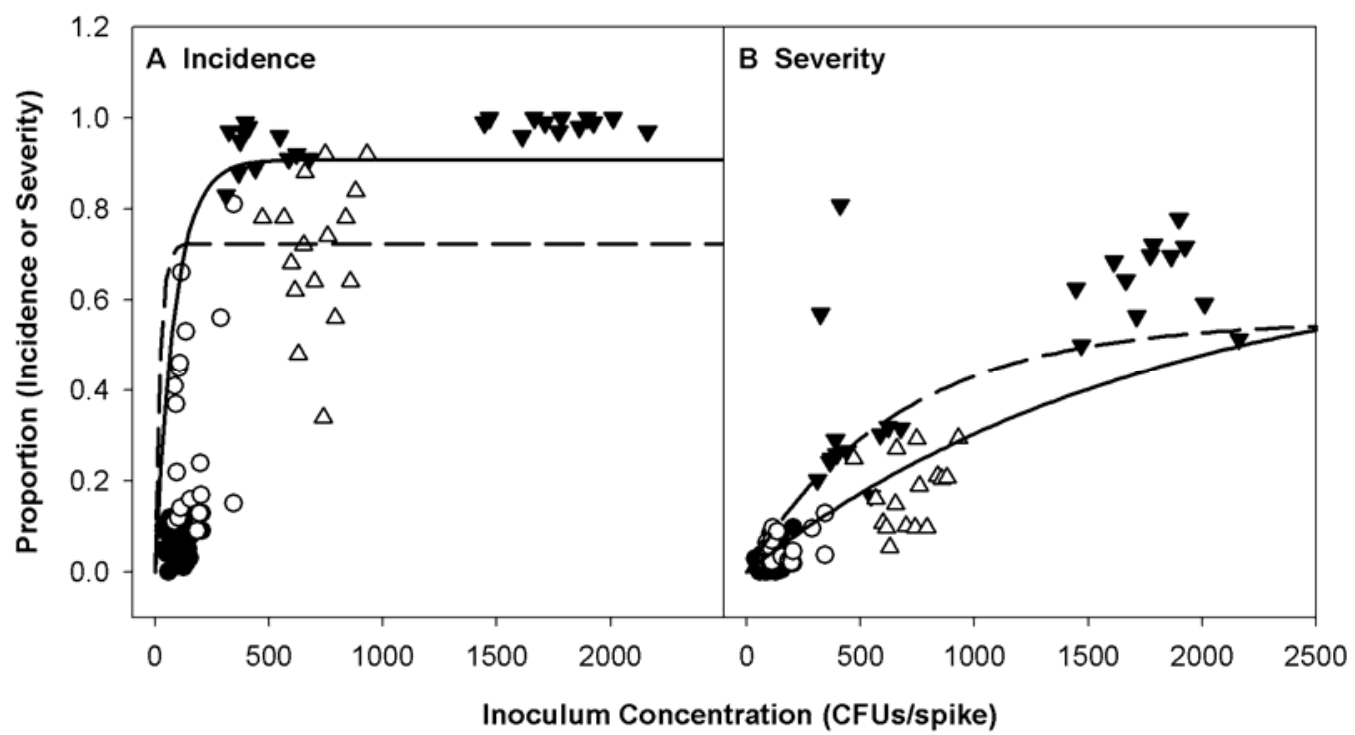

Fig. 3. Mean number of Gibberella zeae CFU per spike for the period of peak flowering in susceptible wheat cultivars versus the resulting disease A, incidence or B, severity for field experiments EDM03 $(\bullet)$, EDM04 $(\boldsymbol{\nabla})$, EDM05 $(\Delta)$, and Residue07 $(O)$. Nonlinear regression lines represent the predicted mean value using the parameter estimates determined with the greenhouse (solid) and field (dashed) inoculum concentration ranges. 
we used the mean daily concentration of $G$. zeae $\mathrm{CFU}$ on wheat spikes during the period of active flowering for an experimental plot for the field calculations. This was done because the interactions between spore deposition, spore survival, and subsequent infection are poorly understood under field conditions $(20,22$, $25,27)$. In addition, spikes in a given field plot of wheat are known not to be uniformly susceptible, especially when multiple tillers are present per plant $(3,20)$. In essence, the impact of inoculum present on a spike during individual days when measurements were taken was unknown and could not be meaningfully included in the analyses. In comparison, inoculum in the greenhouse study was applied as a single dose and the spikes inoculated were chosen to be relatively uniform in terms of growth stage and susceptibility. This likely impacted both the efficiency of infection and those biological factors associated with DON biosynthesis and accumulation between the two groups of studies $(2,12)$.

The environmental conditions likely also had an impact on both disease development and DON accumulation. Extended periods of free moisture and moderate temperatures $\left(15\right.$ to $\left.30^{\circ} \mathrm{C}\right)$ are

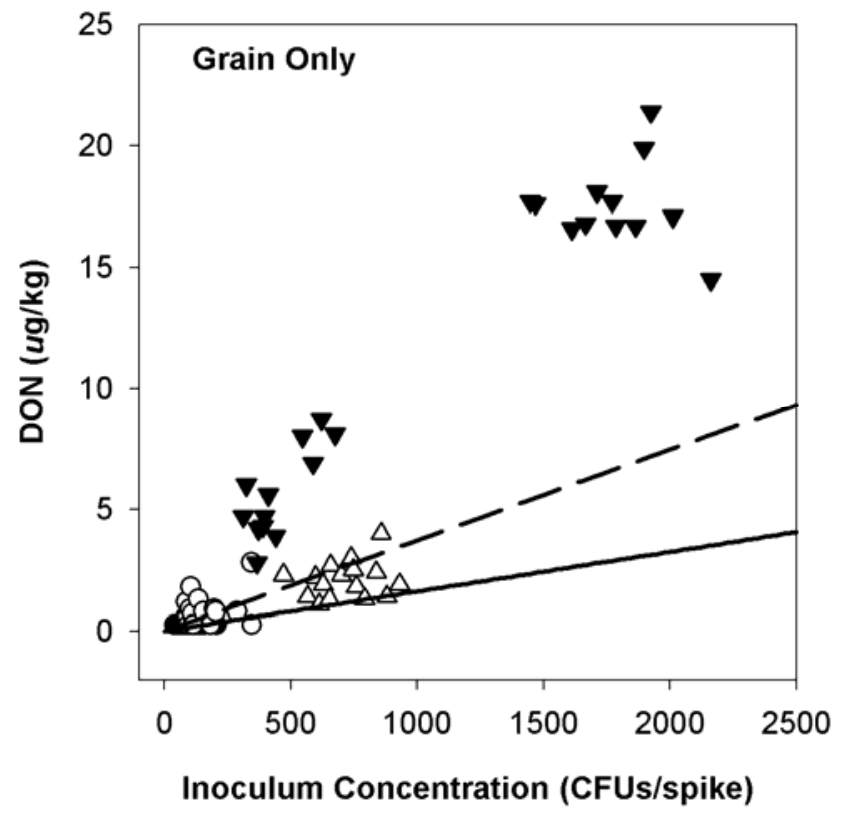

Fig. 4. Mean number of Gibberella zeae CFU per spike for the period of peak flowering in susceptible wheat cultivars versus the resulting deoxynivalenol concentration in the harvested grain for field experiments EDM03 $(\bullet)$, EDM04 ( $)$, EDM05 $(\Delta)$, and Residue07 $(0)$. Linear regression lines represent the predicted mean value using the parameter estimates determined with the greenhouse (solid) and field (dashed) inoculum concentration ranges. known to be required for successful infection by G. zeae $(1,4)$. In the greenhouse study, temperature was always in the optimal range but saturated moisture conditions were only maintained during the infection stage (first $72 \mathrm{~h}$ ). Plants were subsequently kept under conditions of moderate relative humidity (50 to $70 \%$ ) for the remainder of the experiment (data not shown). In the field studies, temperatures and the duration of wetness during the period of highest susceptibility would have been different than in the greenhouse studies and also varied between years. These experimental differences during the infection period for the field studies would have had an impact on both disease development and DON accumulation and added variability to the results that could not be well explained by the models.

Although the field results were not effectively explained by the functions and parameters estimates identified from the greenhouse study, this does not necessarily negate the utility of these models for practical disease or DON prediction. The results of our study support previous research which concluded that disease incidence or severity were related to spike-borne inoculum concentration during the period of peak susceptibility for wheat $(1,4,28)$. Our study also demonstrated that DON accumulation in the grain is positively related to inoculum concentration in both artificial (greenhouse) and field situations. The combination of the models developed in this study with others has the potential to increase the accuracy of current risk-advisory systems for predicting disease severity as well as adding functionality that predicts the risk of economically important DON concentrations.

Related research investigating spike-borne inoculum dynamics has identified environmental factors that explain inoculum concentration in a time-delayed (lag) manner $(19,21)$. If inoculum concentration and the risk of infection on a given day could be accurately predicted, then the models developed from this research could help predict when damaging FHB or DON situations were likely to occur in a susceptible crop. This would provide producers with additional information useful in managing losses from $G$. zeae. For example, if such a system could predict the presence of $>500 G$. zeae propagules per wheat spike (or $>1,200$, depending on which slope was used) and when the environment was conducive for infection, then the model developed in our study would indicate that there was a high risk that a susceptible wheat crop would have a DON concentration $>2 \mu \mathrm{g} / \mathrm{kg}$. This level is often used as an economic threshold for millers and is the type of information that producers could use to make management decisions that minimize losses from FHB.

\section{ACKNOWLEDGMENTS}

This material is based upon work supported in part by the United States Department of Agriculture (USDA) under agreement no. 59-07904-107. This is a cooperative project with the United States Wheat \&

TABLE 2. Fit statistics for the field validations of the greenhouse (GH)-derived models predictive of Fusarium head blight (FHB) incidence and severity and deoxynivalenol (DON) accumulation in the grain-only samples based on Gibberella zeae inoculum load (CFU/spike) as measured on susceptible wheat lines under field conditions

\begin{tabular}{|c|c|c|c|c|c|c|}
\hline \multirow[b]{2}{*}{ Response variable ${ }^{\mathrm{w}}$} & \multirow[b]{2}{*}{ Inoculum $^{\mathrm{x}}$} & \multicolumn{4}{|c|}{ Sum of squares error ${ }^{y}$} & \multirow[b]{2}{*}{ Corr. coeff. $(P$ value $)$} \\
\hline & & EDM03 & EDM04 & EDM05 & Residue07 & \\
\hline \multirow[t]{2}{*}{ Incidence } & $\mathrm{GH}$ & 6.31 & 0.11 & 1.03 & 36.84 & $0.56(<0.001)$ \\
\hline & Field & 3.64 & 1.38 & 0.38 & 25.85 & $0.34(<0.001)$ \\
\hline Severity & Field & 0.12 & 0.74 & 0.60 & 0.28 & $0.85(<0.001)$ \\
\hline \multirow{2}{*}{ DON, grain } & GH & 12.61 & $2,946.12$ & 24.54 & 0.65 & $0.93(<0.001)$ \\
\hline & Field & 7.70 & $1,694.61$ & 17.25 & 2.21 & $0.93(<0.001)$ \\
\hline
\end{tabular}

\footnotetext{
${ }^{w}$ Estimated values for the disease metrics and DON were calculated using the negative exponential and linear functions, respectively. Negative exponential function: $y=L \times\left[1-e^{(-k \times x)}\right]$; linear function: $y=a+b \times x$; in both functions, $x$ is the number of conidia present on the wheat spike.

${ }^{\mathrm{x}}$ Inoculum dose range used to calculate the parameter estimates: 0 to 25,000 and 0 to 2,500 propagules/spike for the GH and field ranges, respectively.

${ }^{y}$ Wheat cvs. Norm and Russ were used for the FHB epidemiology (EDMxx) and residue management (Residue) studies, respectively. The numbers indicate the year in which the study was performed (e.g., EDM05 was in 2005).

z Pearson correlation coefficient and $P$ value between the observed and predicted responses from the corresponding statistical model.
} 
Barley Scab Initiative. Any opinions, findings, conclusions, or recommendations expressed in this publication are those of the authors and do not necessarily reflect the view of the USDA. Additional support was provided by the South Dakota Wheat Commission and South Dakota Agricultural Experiment Station. We acknowledge our collaborators in the field studies (in alphabetical order): T. Adhikari, S. Ali, E. DeWolf, R. Dill-Macky, P. Lipps, L. Madden, P. Paul, G. Shaner, and G. Yuen.

\section{LITERATURE CITED}

1. Andersen, A. L. 1948. The development of Gibberella zeae head blight of wheat. Phytopathology 38:595-611.

2. Beyer, M., Verreet, J. A., and Ragab, W. S. M. 2005. Effect of relative humidity on germination of ascospores and macroconidia of Gibberella zeae and deoxynivalenol production. Int. J. Food Microbiol. 98:233-240.

3. Del Ponte, E. M., Fernandes, J. M. C., and Bergstrom, G. C. 2007. Influence of growth stage on Fusarium head blight and deoxynivalenol production in wheat. J Phytopathol. 155:577-581.

4. De Wolf, E. D., Madden, L. V., and Lipps, P. E. 2003. Risk assessment models for wheat Fusarium head blight epidemics based on within-season weather data. Phytopathology 93:428-435.

5. Dufault, N. S., Dewolf, E. D., Lipps, P. E., and Madden, L. V. 2006. Role of temperature and moisture in the production and maturation of Gibberella zeae perithecia. Plant Dis. 90:637-644.

6. Fernando, W. G. D., Paulitz, T. C., Seaman, W. L., Dutilleul, P., and Miller, J. D. 1997. Head blight gradients caused by Gibberella zeae from area sources of inoculum in wheat field plots. Phytopathology 87:414-421.

7. Francl, L., Bergstrom, G., Gilbert, J., Pedersen, W., Dill-Macky, R., Sweets, L., Corwin, B., Jin, Y., and Gallenberg, D. 1999. Daily inoculum levels of Gibberella zeae on wheat spikes. Plant Dis. 83:662-666.

8. Gilbert, J. 2003. Sources and dispersal of Gibberella zeae/Fusarium graminearum inoculum. Pages 107-112 in: Proc. Third Can. Workshop on Fusarium Head Blight/Colloque Canadien Sur La Fusariose, Winnipeg, MB, Canada.

9. Gilbert, J., and Tekauz, A. 2000. Review: recent developments in research on Fusarium head blight of wheat in Canada. Can. J. Plant Pathol. 22:1-8.

10. Large, E. C. 1954. Growth stages in cereals-illustration of the Feekes scale. Plant Pathol. 3:128-129.

11. Lindstrom, M. J., and Bates, D. M. 1990. Nonlinear mixed effects models for repeated measures data. Biometrics 46:673-687.

12. Magan, N., Hope, R., Colleate, A., and Baxter, E. S. 2002. Relationship between growth and mycotoxin production by Fusarium species, biocides and environment. Eur. J. Plant Pathol. 108:685-690.

13. Markell, S. G., and Francl, L. J. 2003. Fusarium head blight inoculum: Species prevalence and Gibberella zeae spore type. Plant Dis. 87:814-820.

14. McMullen, M. 2003. Impacts of Fusarium head blight on the North American agricultural community: the power of one disease to catapult change. Pages 484-503 in: Fusarium Head Blight of Wheat and Barley. K. J. Leonard and W. R. Bushnell, eds. American Phytopathological Society Press, St. Paul, MN.
15. Nelson, P. E., Toussoun, T. A., and Marasas, W. F. O. 1983. Fusarium species: An illustrated manual for identification. Pennsylvania State University Press, University Park.

16. Nita, M., De Wolf, E., Madden, L., Paul, P., Shaner, G., Adhikari, T., Ali, S., Stein, J., and Osborne, L. 2006. Effect of corn residue level on disease intensity of Fusarium head blight (FHB) and on deoxynivalenol (DON) concentration: A multi-state field study. (Abstr.) Phytopathology 96:S85.

17. Osborne, L., Jin, Y., and Kohl, R. 2000. Fusarium head blight: Inoculum detection, disease progress, and environmental influences. Pages 163-168 in: Proc. 2000 Nat. Fusarium Head Blight Forum, Michigan State University, East Lansing.

18. Osborne, L. E. 2004. Fusarium graminearum mycotoxin levels in spring wheat with varying amounts of corn residue. (Abstr.) Phytopathology 94:S79.

19. Osborne, L. E., and Stein, J. M. 2006. Temporal inoculum dynamics for Fusarium head blight of wheat and barley in South Dakota. (Abstr.) Phytopathology 96:S88.

20. Osborne, L. E., and Stein, J. M. 2007. Epidemiology of Fusarium head blight on small-grain cereals. Int. J. Food Microbiol. 119:103-108.

21. Paul, P. A., Lipps, P. E., Dewolf, E., Shaner, G., Buechley, G., Adhikari, T., Ali, S., Stein, J., Osborne, L., and Madden, L. V. 2007. A distributed lag analysis of the relationship between Gibberella zeae inoculum density on wheat spikes and weather variables. Phytopathology 97:1608-1624.

22. Paul, P. A., Lipps, P. E., and Madden, L. V. 2005. Relationship between visual estimates of Fusarium head blight intensity and deoxynivalenol accumulation in harvested wheat grain: A meta-analysis. Phytopathology 95:1225-1236.

23. Paulitz, T. C. 1996. Diurnal release of ascospores by Gibberella zeae in inoculated wheat plots. Plant Dis. 80:674-678.

24. R Development Core Team. 2007. R: a language and environment for statistical computing. R Foundation for Statistical Computing, Vienna.

25. Rossi, V., Ravanetti, A., Pattori, E., and Giosue, S. 2001. Influence of temperature and humidity on the infection of wheat spikes by some fungi causing Fusarium head blight. J. Plant Pathol. 83:189-198.

26. Schmale, D. G., III, Shah, D. A., and Bergstrom, G. C. 2005. Spatial patterns of viable spore deposition of Gibberella zeae in wheat fields. Phytopathology 95:472-479.

27. Schmale, D. G., III, Shields, E. J., and Bergstrom, G. C. 2006. Night-time spore deposition of the Fusarium head blight pathogen, Gibberella zeae, in rotational wheat fields. Can. J. Plant Pathol. 28:100-108.

28. Stack, R. W. 1989. A comparison of the inoculum potential of ascospores and conidia of Gibberella zeae. Can. J. Plant Pathol. 11:137-142.

29. Stack, R. W., and McMullen, M. P. 1985. Head blighting potential of Fusarium species associated with spring wheat heads. Can. J. Plant Pathol. 7:79-82.

30. Stein, J. M., Osborne, L., and Glover, K. 2008. Fusarium head blight severity and deoxynivalenol accumulation in wheat spike tissues as a function of Gibberella zeae inoculum density. (Abstr.) Phytopathology 98:S150.

31. Sutton, J. C. 1982. Epidemiology of wheat head blight and maize ear rot caused by Fusarium graminearum. Can. J. Plant Pathol. 4:195. 